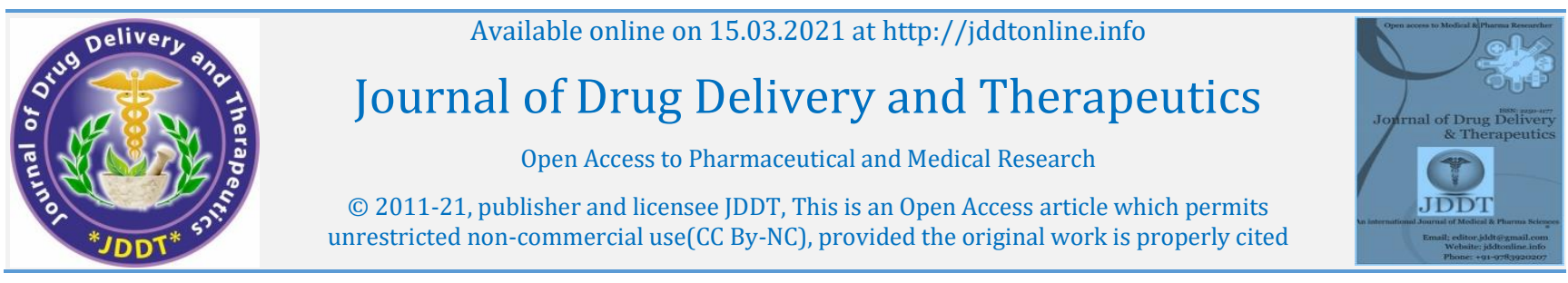

Open Access Full Text Article

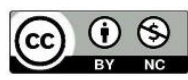

Review Article

\title{
Case studies of enhanced pharmacodynamic activity of poorly oral bioavailable drugs via solid lipid nanoparticles
}

\author{
Raju D* \\ Prathibha Pharmacy College, Warangal, Telangana, India
}

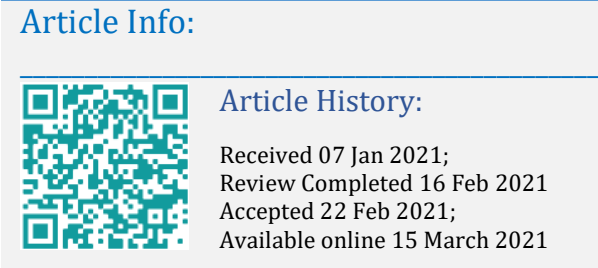

Cite this article as:

Raju D, Case studies of enhanced pharmacodynamic activity of poorly oral bioavailable drugs via solid lipid nanoparticles, Journal of Drug Delivery and Therapeutics. 2021; 11(2):204-208

DOI: http://dx.doi.org/10.22270/jddt.v11i2.4582

*Address for Correspondence:

Raju D, Prathibha Pharmacy College, Warangal,

Telangana, India

\begin{abstract}
Solid lipid nanoparticles (SLNs) considered as an alternative vehicle for the enhanced oral absorption of drugs, and also to enhance therapeutic effectiveness after oral administration. Pharmacodynamic activity of drug is mainly describes the pharmacological and therapeutic activity of drug to the biological system. Lipid nanoparticles especially SLNs made of physiological inert lipid molecules and helps the lymphatic transport. Numerous literatures is available on the effect of SLNs and other colloidal carrier systems on the pharmacokinetic activity of poorly bioavailable drugs, to improve their oral absorption and also respective mechanisms for the improved oral bioavailability. However, very few literatures is reported on the pharmacodynamic activity and the effect of dose on the pharmacodynamic activity. Therefore, the current review is mainly dealing with the effect of SLNs on the pharmacodynamic activity discussed.
\end{abstract}

Keywords: Oral absorption, solid lipid nanoparticles, lymphatic transport, pharmacokinetics, pharmacodynamics.

\section{INTRODUCTION}

In general, drugs with low water solubility, difficulty in formulation development as conventional dosage forms, as they existent problems such as slow onset of action, low oral bioavailability, shortage of dose proportionality, failure to attain stable plasma levels (steady state concentration), and dislikable side effects ${ }^{1-4}$. New approaches are developed and reported to enhance the oral bioavailability therapeutic efficiency by gastro retentive delivery-10, through permeation by buccal delivery ${ }^{11-14}$, solubility by complexation and liquisolid comapcts $15-17$, osmotic drug delivery ${ }^{18,19}$, transfersomes ${ }^{20,21}$, colon delivery ${ }^{22}$, alternative delivery systems ${ }^{23,24}$ etc. Administration by the oral route of the drug is most prevalent strategy for drug transport. Regardless of the fame and flexibility of the oral course, huge issues endure. Not all drugs have the physical, chemical or biological qualities essential for the effective treatment by oral delivery 25 . Issues, for example, poor solubility or chemical stability of the gastrointestinal tract, poor porousness through the organic films or affectability to digestion are outstanding to bring about the dismissal of potential medication hopefuls as useful items ${ }^{26,27}$. Lipid based drug delivery have been proposed as a method for bypassing some of safer drugs or physical obstructions related with inadequately effectiveness of drugs 28,29 . These potential drug deliveries incorporate the more traditional structures, for example, emulsions and microemulsions, and in addition later ones, for example, liposomes, microspheres, solid lipid nanoparticles (SLNs) ${ }^{30-33}$ and nanostructured lipid carriers (NLC) ${ }^{34-38}$ and also reduction of particle size (nanosuspension) ${ }^{39-41}$ to improve the oral bioavailability.

Lipid nanoparticles such as SLNs are emerging as alternative carriers to colloidal drug systems, for controlled systems and targeted delivery. These are in submicron size range (50$1000 \mathrm{~nm}$ ) and are made of biocompatible and biodegradable materials capable of incorporating both lipophilic, hydrophilic drugs, protein delivery 42,43 and also surface modification for enhanced delivery and stability ${ }^{44}$. SLNs combine the advantage of different colloidal carriers, for instance, like emulsions and liposomes, these are physiologically acceptable and like polymeric nanoparticles, controlled release of drug from lipid matrix can be anticipated 45,46 .

SLNs are particles made from solid lipids (i.e., lipids solid at room temperature and also at body temperature) and stabilized by surfactant(s). By definition, the lipids can be highly purified triglycerides, complex glyceride mixtures or even waxes. Through the work of various research groups, the SLN carrier system has been characterized intensively in various applications also ${ }^{47-51}$.

Great progress has been made in the treatment of a variety of diseases by using drug delivery systems including solid lipid nanoparticles (SLN). Controlled drug delivery, enhancement of bioavailability of entrapped drugs via modification of dissolution rate r2-54 $^{2}$ and/or improvement of tissue 
distribution and targeting of drugs ${ }^{55-58}$ by using SLNs and NLCs have been reported.

Extensive literature was reported in the development of drug loaded SLNs and NLCs to improve the oral bioavailability of poorly soluble and absorbable drugs ${ }^{59-62}$. But, there is no specific review reported on the pharmacodynamic activity of SLNs reported. Therefore, in this review, mainly discuss about the pharmacodynamic effect of drug loaded SLNs of low bioavailability drugs.

\section{CASE STUDIES}

\section{Simvastatin solid lipid nanoparticles}

Simvastatin (ST) is a lipid lowering drug with low oral bioavailability of about $5 \%$. The poor bioavailability constraint requires the development of efficient delivery system, which could improve the oral absorption and transport of ST. ST-SLNs are prepared using homogenization method, employed Compritol and Precirol as solid lipids, Tween 80 as surfactant. The mean particle size, zeta potential (ZP), entrapment efficiency (EE) was observed to be below $200 \mathrm{~nm}, 96 \%$ and $-25 \mathrm{mV}$ respectively. The pharmacodynamic study of ST-SLNs is conducted in wistar rats at a dose of $0.1 \mathrm{~mL}$ in $1 \mathrm{~g} / \mathrm{kg}$ of poloxamer $\mathrm{F}-127$ solution induced hyperlipedemia. From the results, the formulation of ST reduced the total cholesterol, low density lipoproteins and increased high density lipoproteins compared with drug suspension 63 . List of various drugs loaded SLNs on pharmacodynamic activity showed in Table 1.

Table 1: List of drugs loaded solid lipid nanoparticles on pharmacodynamic effect up on oral administration

\begin{tabular}{|l|l|l|c|}
\hline Drug & Animal & Inference & Ref \\
\hline Simvastatin & Female Sprague Dawley rats & Decrease in TC, LDL and increase in HDL & 63 \\
\hline Nisoldipine & Wistar male rats & Reduced systolic blood pressure upto 36 h & 64 \\
\hline Galantamine & Rats & Memory restoration & 66 \\
\hline Candesartan cilexetil & Wistar male rats & Systolic blood pressure decreased upto $48 \mathrm{~h}$ & 67 \\
\hline $\begin{array}{l}\text { Dexamethasone- cholesteryl } \\
\text { butyrate }\end{array}$ & Mice & additive anti-inflammatory & 68 \\
\hline Isradipine & Wistar male rats & Reduced blood pressure & 70 \\
\hline Rosuvastatin calcium & Rats & Reduced TC and LDL levels, increased HDL levels & 69 \\
\hline
\end{tabular}

\section{Nisoldipine solid lipid nanoparticles}

Kishan and his team mainly research focus on the pharmacokinetic and pharmacodynamic effect of drug loaded SLNs, SEDDS and nanoemulsions to tumor targeting. Same group also reported the nanodelivery systems of some drugs to improve the oral bioavailability 64 . Initially, Nisoldipine (ND) loaded SLNs were developed and reported. ND is a calcium channel antagonist, used for the treatment of hypertension. The oral bioavailability of ND is only about $5 \%$ due to poor water solvency and also presystemic metabolism in gut wall. Therefore, an attempt was made to develop ND loaded SLNs (ND-SLNs) to enhance oral absorption using central composite design. ND-SLNs showed mean particle size of below $100 \mathrm{~nm}$, PDI of 0.23 , ZP of -25 $\mathrm{mV}$ and $\mathrm{EE}$ of 91\%. Pharmacodynamic studies were conducted in male wistar rats in fructose induced hypertensive rats. The rats treated with $10 \mathrm{mg} / \mathrm{kg}$ of statistically optimized ND-SLNs in comparison with pure drug suspension. Reduced systolic blood pressure was observed up to $36 \mathrm{~h}$ in SLN treated group, whereas suspension group showed up to $12 \mathrm{~h}$. Therefore, the bioavailability of ND was improved by using SLNs delivery system $^{65}$ (Narendar and Kishan, 2015).

\section{Galantamine solid lipid nanoparticles}

Medhi et al., investigated Galant amine loaded solid lipid nanoparticles (GM-SLNs) to improve the oral bioavailability and also memory function in amnesia induced mice ${ }^{66}$. GMSLNs were prepared by employing microemulsion method. Prepared SLNs have the particle size of below $100 \mathrm{~nm}$ with EE $83.4 \pm 0.6 \%$. Further, pharmacodynamic activity of the GM-SLNs were evaluated for brain targeting and memory enhancement in isoproterenol-induced amnesic rats using Morris water maze test. From the pharmacodynamic results, noticed that enhanced or restoration of cognition function in memory loss rats, which is statistically significant.

\section{Candesartan cilexetil solid lipid nanoparticles}

Dudhipala and Veerabrahma, developed the candesartan cilexetil (CC) loaded solid lipid nanoparticles to prolong the drug release with improved pharmacokinetic and pharmacodynamic activity ${ }^{67}$. CC belongs to BCS class II drug with poor oral bioavailability, hence, prone to improve the oral transportation using SLNs delivery system. CC-SLNs were prepared using hot homogenization coupled with sonication technique. Solid lipids, surfactant and cosurfactnt selected for the making of SLNs are triglycerides (dynasan 114, 116 and 118), egg lecithin and poloxamer 188 respectively. From the physical characteristics and in vitro release studies, optimized SLNs were identified. DSC and XRD studies revealed the conversion of crystalline to amorphous form of CC in SLN formulation. Particle size of the SLNs was nearly spherical in shape with increased polydispersity, performed by SEM and TEM analysis. Pharmcodynamic study of SLNs are conducted in fructose $(10 \% \mathrm{w} / \mathrm{v})$ induced hypertensive wistar rats at a dose of 10 $\mathrm{mg} / \mathrm{kg}$. CC-SLNs showed reduction blood pressure immediately and continued for up to $48 \mathrm{~h}$. Hence, the results successfully demonstrate the role of SLNs as oral bioavailability enhanced carrier.

\section{Dexamethasone cholesteryl butyrate solid lipid nanoparticles}

Combination of dexamethasone cholesteryl butyrate loaded solid lipid nanoparticles (DC-SLNs) were prepared and characterized for enhanced ant-inflammatory activity. DCSLNs were prepared by hot micro emulsion method. The pharmacodynamic activity of SLNs is tested by $4 \%(\mathrm{w} / \mathrm{v})$ dextran sulfate sodium salt in mice. From the results, DC 
nanoformulation administration was able to achieve a significant cytokine decrease compared to the cytokine plasma concentration of the untreated mice with dextran sulfate sodium-induced colitis. Specifically, DC-SLN induced a IL-1 $\beta$ plasma concentration of $61.77 \% \pm 3.19 \%$, whereas D or C used separately induced a concentration of $90.0 \%$ $\pm 2.8 \%$ and $91.40 \% \pm 7.5 \%$, respectively; DC-SLN induced a TNF- $\alpha$ plasma concentration of $30.8 \% \pm 8.9 \%$, whereas D or C used separately induced ones of $99.5 \% \pm 4.9 \%$ and $71.1 \%$ $\pm 10.9 \%$, respectively 68 .

\section{Rosuvastatin calcium}

Narendar and Kishan, also studied the effect of rosuvastatin calcium (RC) loaded SLNs on pharmacokinetic and pharmcodynaimc activity, as a vehicle for improved oral absorption ${ }^{69}$. RC-SLNs were prepared with dynasan 112, stearic acid and glyceryl monostearte. Previously, RC-SLNs also developed with triglycerides (dynasan 114, 116 and $18)^{6}$. In order to reduce the manufacturing cost, monoglycerides and triglyceride with low cost were used. RC-SLNs with dynasan 112 showed particle size, PDI, ZP and EE of below $75 \mathrm{~nm}, 0.23,-26 \mathrm{mV}$ and 93\%, respectively. Pharmcodynamic studies were conducted in wistar rats at a dose of $10 \mathrm{mg} / \mathrm{kg}$. The hyperlipidemia in rats was induced by intraperitoneal injection of Triton-X-100 (100 mg/kg) in normal saline solution. RC-SLNs showed statistically significant reduction in the serum total cholesterol, low density lipoproteins, and slight increase in high density lipoproteins. These levels of changes were continued for 36 $\mathrm{h}$ and $24 \mathrm{~h}$ in RC-SLNs and RC suspension treated group respectively. Thus, $\mathrm{RC}$ oral bioavailability was enhanced by making in to SLN delivery system.

\section{Isradipine solid lipid nanoparticles}

Isradipine (ID) have the oral bioavailability of about $5 \%$ due to poor aqueous solubility and chemical instability. Therefore, SLNs of ID were developed to enhance the oral transport by avoiding first-pass metabolism too. ID-SLNs were prepared with probe sonication method. Prepared SLNs evaluated for optimized system with good stability properties. Pharmacodynamic studies in rats exhibited declined levels of systolic blood pressure in fructose induced hypertension model 70 .

\section{Conclusion and future prospective}

Solid lipid nanoparticles are very multifaceted structure with distinguishable advantages and disadvantages related to other colloidal carriers. Due to the stability and drug expulsion problems of SLNs, the NLCs were emerge., SLNs offering virtuous release profile and it is made them widespread in nano pharmaceutical research field and other applications such as food and bioactive delivery. SLNs are produced with stable and reproducible properties by using known techniques. SLNs provides the enhanced pharmacodynamic effect in the preclinical studies in poorly oral absorbable drugs. The research on going on SLNs as well as of commercialization is possible and practicable in the laboratory and on the large scale. Additional efforts are needed to confirm the pharmacokinetic and pharmacodynamics of SLNs in human population and also in clinical point of view.

\section{DECLARATION OF INTEREST}

Author declares no conflict of interest.

\section{REFERENCES}

1. Sastry SV, Nyshadham JR, Fix JA, Recent technological advances in oral drug delivery-a review, Pharmaceutical science and technology today, 2000; 3(4):138-45.
2. Andrew JH, William NC, Lipid-based vehicles for the oral delivery of poorly water-soluble drugs, Advanced Drug Delivery Reviews, 1997; 25:103- 128.

3. Alekya T, Narendar D, Mahipal D, Arjun N, Nagaraj B, Design and evaluation of chronomodulated drug delivery of tramadol hydrochloride, Drug research, 2018; 68(03):174-80.

4. Müller RH. Colloidal carriers for controlled drug delivery and targeting: Modification, characterization and in vivo distribution, Taylor \& Francis; 1991.

5. Narendar D, Palem CR, Reddy S, Rao YM, Pharmaceutical development and clinical pharmacokinetic evaluation of gastro retentive floating matrix tablets of levofloxacin, International Journal Pharmaceutical Sciences and Nanotechnology, 2011; 4(3):1461-1467.

6. Donthi MR, Dudipala N, Komalla DR, Suram D, Banala N, Design and evaluation of floating multi-unit mini tablets (mumts) muco adhesive drug delivery system of famotidine to treat upper gastro intestinal ulcers. Journal of Pharmacovigilance, 2015; Oct 12.

7. Narendar D, Chinna Reddy P, Sunil R, Madhusudan Rao Y, Development of floating matrix tablets of Ofloxacin and Ornidazole in combined dosage form: in vitro and in vivo evaluation in healthy human volunteers, International Journal of Drug Delivery, 2012; 4:462-469.

8. Donthi MR, Dudhipala NR, Komalla DR, Suram D, Banala N. Preparation and Evaluation of Fixed Combination of Ketoprofen Enteric Coated and Famotidine Floating Mini Tablets by Single Unit Encapsulation System. Journal of Bioequivalence \& Bioavailability. 2015; 7(6):279.

9. Narendar D, Arjun N, Karthik Yadav J, Ramesh B, Amoxycillin trihydrate floating-bioadhesive drug delivery system for eradication of helicobacter pylori: preparation, in vitro and ex vivo evaluation, Journal of bioequvilance bioavailability, 2016; 8(3):118-124.

10. Narendar D, Someshwar K, Arjun N, Madhusudan Rao Y,. Quality by design approach for development and optimization of Quetiapine Fumarate effervescent floating matrix tablets for improved oral delivery, Journal of Pharmaceutical investigation, 2016; 46(3):253-263.

11. Chinna Reddy Palem, Narendar D, Sunil Kumar Battu, Michael A. Repka, Madhusudan Rao Y, Development, optimization and in vivo characterization of domperidone controlled release hot melt extruded films for buccal delivery. Drug Development and Industrail Pharmacy, 2016, 42(3):473-484.

12. Chinna Reddy P Narendar D, Sunil Kumar B, Satyanarayana G, Madhusudan Rao Y, Combined dosage form of pioglitazone and felodipine as mucoadhesive pellets via hot melt extrusion for improved buccal delivery with application of quality by design approach, Journal of drug delivery science and technology, 2015; 30:209-219.

13. Banala N, Peddapalli $\mathrm{H}$, Dudhipala N, Chinnala KM, Transmucosal delivery of duloxetine hydrochloride for prolonged release: preparation, in vitro, ex vivo characterization and in vitro-ex vivo correlation. International Journal of Pharmaceutical Sciences and Nanotechnology. 2018; 11(5):4249-58.

14. Palem CR, Ramesh G, Narender D, Vamshi Vishnu Y, Madhusudan Rao Y, Transmucosal delivery of domperidone from bilayered buccal patches: in vitro, ex vivo and in vivo characterization, Arch pharmacal research, 2011; 34(10):17011710.

15. Swetha E, Narendar D, Influence of $\beta$-Cyclodextrin and hydroxypropyl- $\beta$-cyclodextrin on enhancement of solubility and dissolution of isradipine, International Journal Pharmaceutical Sciences and Nanotechnology, 2017; 10(3):3752-3757.

16. Palem CR, Reddy ND, Satyanarayana G, Varsha BP, Development and optimization of Atorvastatin calcium-cyclodextrin inclusion complexed oral disintegrating tablets for enhancement of solubility, dissolution, pharmacokinetic and pharmacodynamic activity by central composite design, International Journal Pharmaceutical Sciences and Nanotechnology, 2016; 9(2):1-11.

17. Butreddy A, Narendar D, Enhancement of solubility and dissolution rate of trandolapril sustained release matrix tablets by liquisolid compact approach, Asian Journal of Pharmaceutics, 2015; 9(4):290-297.

18. Narendar D, Arjun N, Sunitha K, Harika K, Nagaraj B, Development of osmotically controlled oral drug delivery systems of tramadol hydrochloride: effect of formulation 
variables on in-vitro release kinetics, Asian Journal of Pharmaceutics, 2016; 10(3):1-10.

19. Arjun N, Narendar D, Sunitha K, Harika K, Madhusudan Rao Y, Nagaraj B, Development, evaluation and influence of formulation and process variables on in vitro performance of oral elementary osmotic device of atenolol, International Journal of Pharmaceutical Investigation, 2016; 6(4):1-9.

20. Pitta SK, Dudhipala N, Narala A, Veerabrahma K, Development and evaluation of zolmitriptan transfersomes by Box-Behnken design for improved bioavailability by nasal delivery, Drug Development and Industrial Pharmacy, 2018; 44(3):484-492.

21. Narendar D, Riyaz PMD, Ahmed AY, Nagaraj B, Effect of lipid and edge activator concentration on development of Aceclofenac loaded transfersomes gel for transdermal application: in vitro and ex vivo skin permeation, Drug Development and Industrial Pharmacy, 2020; 46(8):1334-1344.

22. Rajitha R, Narendar D, Arjun N, Mahipal D, Nagaraj B. Colon delivery of naproxen: preparation, characterization and in vivo evaluation, International Journal Pharmaceutical Sciences and Nanotechnology, 2016; 9(3): 1-10.

23. Nagaraj B, Anusha K, Narendar D, Sushma P, Formulation and evaluation of microemulsion-based transdermal delivery of duloxetine hydrochloride, International Journal of Pharmaceutical Sciences and Nanotechnology, 2020; 13(1):4773-82.

24. Shruthi K, Narendar D, Arjun N, Kishan V, Development and Antimicrobial Evaluation of Binary Ethosomal Topical Gel of Terbinafine Hydrochloride for the Treatment of Onychomycosis, International Journal Pharmaceutical Sciences and Nanotechnology, 2018; 11:3998-4005.

25. Narendar D, Arjun N, Ramesh B, Recent Updates in the formulation strategies to enhance the bioavailability of drugs administered via intranasal route, Journal of bioequivalence and bioavailability, 2016; 8(5):204-207.

26. Bruce JA, Novel formulation strategies for improving oral bioavailability of drugs with poor membrane permeation or presystemic metabolism, Journal of Pharmaceutical scienecs, 1993; 82(10):979-987.

27. Doodipala $\mathrm{R}$, A review of novel formulation strategies to enhance oral delivery of zaleplon, Journal of bioequivalence and bioavailability, 2016; 8(5):211-213.

28. Mehnert W, Mäder K, Solid lipid nanoparticles production, characterization and applications, Advanced Drug Delivery Reviews, 2012; 64:83-101.

29. Dudhipala N, Influence of solid lipid nanoparticles on pharmacodynamic activity of poorly oral bioavailable drugs, International Journal of Pharmaceutical Sciences and Nanotechnology, 2020; 13(4):4979-83.

30. Müller RH, Mäder K, Gohla S, Solid lipid nanoparticles (SLN) for controlled drug delivery a review of the state of the art, European Journal of Pharmaceutics and Biopharmacokinetics, 2000; 50:161-177.

31. Banala N, Tirumalesh C, Suram D, Dudhipala N, Zotepine loaded lipid nanoparticles for oral delivery: preparation, characterization, and in vivo pharmacokinetic studies, Future Journal of Pharmaceutical Sciences, 2020; 6(1):37.

32. Wissing SA, Kayser O, Muller RH, Solid lipid nanoparticles for parenteral drug delivery, Advanced Drug Delivery Reviews, 2004; 56:1257-72.

33. Banala N, Cernam T, Suram D, Dudhipala N, Design, development and in vivo pharmacokinetic evaluation of zotepine loaded solid lipid nanoparticles for enhanced oral bioavailability, ACTA Pharmaceutica Sciencia, 2020.

34. Schwarz C, Solid lipid nanoparticles (SLN) for controlled drug delivery II. Drug incorporation and physicochemical characterization, Journal of Microencapsulation, 1999; 16(2):205-213.

35. Narendar D, Karthik Yadav J, Thirupathi G, Comparative study of nisoldipine-loaded nanostructured lipid carriers and solid lipid nanoparticles for oral delivery: preparation, characterization, permeation and pharmacokinetic evaluation, Artificial cells, nanomedicine biotechnology, 2018; 46(S2):616-625.

36. Tirumalesh C, Suram D, Dudhipala N, Banala N, Enhanced pharmacokinetic activity of Zotepine via nanostructured lipid carrier system in Wistar rats for oral application, Pharmaceutical Nanotechnology, 2020; 8(2):158-160.

37. Suvarna G, Narender D, Kishan V, Preparation, characterization and in vivo evaluation of rosuvastatin calcium loaded solid lipid nanoparticles, International Journal Pharmaceutical Sciences and Nanotechnology, 2015; 8(1):2779-2785.

38. Ahmed AAY, Narendar D, Mujumdar S, ciprofloxacin loaded nanostructured lipid carriers incorporated into in-situ gels to improve management of bacterial endophthalmitis, Pharmaceutics, 2020; 12(6):572.

39. Arun B, Narendar D, Kishan V, Development of olmesartan medoxomil lipid based nanoparticles and nanosuspension: Preparation, characterization and comparative pharmacokinetic evaluation, Artificial cells, nanomedicine biotechnology, 2018; 46(1):126-137.

40. Nagaraj K, Narendar D, Kishan V, Development of olmesartan medoxomil optimized nanosuspension using Box-Behnken design to improve oral bioavailability, Drug Development and Industrial Pharmacy, 2017; 43(7):1186-1196.

41. Karri V, Butreddy A, Narender D, Fabrication of efavirenz freeze dried nanocrystals: formulation, physicochemical characterization, in vitro and ex vivo evaluation, Advanced Science, Engineering and Medicine, 2015; 7(5):385-392.

42. Almeida AJ, Eliana S, Solid lipid nanoparticles as a drug delivery system for peptides and proteins, Advanced Drug Delivery Reviews, 2007; 59(6):478-490.

43. Narendar D, A comprehensive review on solid lipid nanoparticles as delivery vehicle for enhanced pharmacokinetic and pharmacodynamic activity of poorly soluble drugs. International Journal Pharmaceutical Sciences and Nanotechnology, 2019; 12(2):4421-4440.

44. Luo Y, Teng Z, Li Y, Wang Q, Solid lipid nanoparticles for oral drug delivery: chitosan coating improves stability, controlled delivery, mucoadhesion and cellular uptake, Carbohydrate polymers, 2015; 122:221-229.

45. Mehnert W, Mäder K, Solid lipid nanoparticles production, characterization and applications, Advanced Drug Delivery Reviews, 2001; 47:165-196.

46. Müller RH, Schwarz C, Zur Muhlen A, et al, Incorporation of lipophilic drugs and drug release profiles of solid lipid nanoparticles (SLN). Proc Int Symp Control Rel Bioact Mate, 1994; 21:146-7.

47. Tatke A, Dudhipala $\mathrm{N}$, Janga $\mathrm{KY}$, et al, In situ gel of triamcinolone acetonide-loaded solid lipid nanoparticles for improved topical ocular delivery: tear kinetics and ocular disposition studies, Nanomaterials (Basel), 2019; 27:9(1).

48. Müller RH, Mehnert W, Lucks JS, Schwarz C, Zur Mühlen A, Weyhers H, Freitas C, Ruhl D, Solid lipid nanoparticles (SLN) An alternative colloidal carrier system for controlled drug delivery, European Journal of Pharmaceutics and Biopharmacokinetics, 1995; 41:62-69.

49. Usha KG, Narendar D, Veerabrahma K, Preparation, characterization and in vivo evaluation of felodipine solid lipid nanoparticles to improve the oral bioavailability, International Journal Pharmaceutical Sciences and Nanotechnology, 2015; 8 (4):1-8.

50. Müller RH, Maaûen, S Weyhers H, et al, Cytotoxicity of magnetite loaded polylactide, polylactide/glycolide particles and solid lipid nanoparticles (SLN), International Journal of Pharmaceutics, 1996;138:85- 94.

51. Sandeep V, Arjun N, Kishan V, Lacidipine loaded solid lipid nanoparticles for oral delivery: Preparation, characterization and in vivo evaluation, International Journal Pharmaceutical Sciences and Nanotechnology, 2016; 9(6):3524-30.

52. Venkateswarlu V, Manjunath K, Preparation, characterization and in vitro release kinetics of clozapine solid lipid nanoparticles, Journal of Controlled Release, 2004; 95:627-38.

53. Dudhipala N, Ahmed AAY, Nagaraj B, Colloidal lipid nanodispersion enriched hydrogel of antifungal agent for management of fungal infections: comparative in-vitro, ex-vivo and in-vivo evaluation for oral and topical application, Chemistry and Physics of Lipids, 2020: 104981.

54. Dudhipala N, Ay AA, Amelioration of ketoconazole in lipid nanoparticles for enhanced antifungal activity and bioavailability through oral administration for management of fungal infections, Chemistry and Physics of Lipids, 2020; 232:104953.

55. Göppert TM, Müller RH, Polysorbate-stabilized solid lipid nanoparticles as colloidal carriers for intravenous targeting of drugs to the brain: Comparison of plasma protein adsorption patterns, Journal of Drug Targeting, 2005; 13(3):179-187. 
56. Narendar D, Govardhan K, Capecitabine lipid nanoparticles for anti-colon cancer activity in 1, 2-dimethylhydrazine induced colon cancer: Preparation, cytotoxic, pharmacokinetic and pathological evaluation, Drug Development and Industrial Pharmacy, 2018; 44(10):1572-1582.

57. Uner M, Wissing SA, Yener G, et al, Investigation of skin moisturizing effect and skin penetration of ascorbyl palmitate entrapped in solid lipid nanoparticles (SLN) and nanostructured lipid carriers (NLC) incorporated into hydrogel, Pharmazie, 2005b; 60:751-5.

58. Narendar D, Karthik J, Lipid nanoparticles of zaleplon for improved oral delivery by Box-Behnken design: Optimization, in vitro and in vivo evaluation, Drug Development and Industrail Pharmacy, 2017; 43(7):1205-1214.

59. Zariwala MG, Elsaid N, Jackson TL, et al,). A novel approach to oral iron delivery using ferrous sulphate loaded solid lipid nanoparticles, International Journal of Pharmaceutics, 2013; 456(2):400-407.

60. Pandita D, Kumar S, Poonia N, Lather V, Solid lipid nanoparticles enhance oral bioavailability of resveratrol, a natural polyphenol, Food Research International, 2014; 62:1165-1174.

61. Dudhipala N, Gorre T, Neuroprotective effect of ropinirole lipid nanoparticles enriched hydrogel for parkinson's disease: In vitro, ex vivo, pharmacokinetic and pharmacodynamic evaluation, Pharmaceutics, 2020; 12(5):448.

62. Ji H, Tang J, Li M, Ren J, Zheng N, Wu L, Curcumin-loaded solid lipid nanoparticles with Brij78 and TPGS improved in vivo oral bioavailability and in situ intestinal absorption of curcumin, Drug Delivery, 2016; 23:459-470.
63. Padhye SG, Mangal SN, Simvastatin Solid lipid nanoparticles for oral delivery: formulation development and in vivo evaluation, Indian Journal of Pharmaceutical Sciences, 2013; 75(5):591598.

64. Narendar D, Kishan V, Pharmacokinetic and pharmacodynamic studies of nisoldipine loaded solid lipid nanoparticles by central composite design. Drug Development and Industrial Pharmacy, 2015; 41(12):1968-1977.

65. Narendar D, Kishan V, Candesartan cilexetil nanoparticles for improved oral bioavailability, Therapeutic delivery, 2017; 8(2):79-88.

66. Medhi B, Misra S, Sinha VR, Modi M, Galantamine-loaded solid lipid nanoparticles: Preparation, characterization, and pharmacodynamics evaluations, Alzheimer's and Demen, 2015; 11(7):840-890.

67. Narendar D, Kishan V, Candesartan cilexetil loaded solid lipid nanoparticles for oral delivery: characterization, pharmacokinetic and pharmacodynamic evaluation, Drug Delivery, 2016; 23(2):395-404.

68. Chiara D, Federica F, Benedetta F, Arianna CR, et al, Solid lipid nanoparticles delivering anti-inflammatory drugs to treat inflammatory bowel disease: TEMP effects in an in vivo model, World Journal of gastroenterology, 2017; 23(23):4200-4210.

69. Narendar D, Kishan V, Improved anti-hyperlipidemic activity of Rosuvastatin Calcium via lipid nanoparticles: pharmacokinetic and pharmacodynamic evaluation. European Journal of Pharmaceutics and Biopharmacokinetics, 2017; 110(1):47-57.

70. Thirupathi G, Swetha E, Narendar D, (2017). Role of isradipine loaded solid lipid nanoparticles in the pharmacodynamic effect of isradipine in rats, Drug research, 2017; 67(03):163-169. 